\title{
Frustrated Blume-Emery-Griffiths model
}

\author{
Georg R. Schreiber* \\ Service de Physique Théorique, C.E.A.-Saclay, F - 91191 Gif-sur-Yvette Cedex France \\ and Division de Physique Théorique, Institut de Physique Nucléaire \\ Université Paris-Sud F-91406 Orsay Cedex France
}

\begin{abstract}
A generalised integer S Ising spin glass model is analysed using the replica formalism. The bilinear couplings are assumed to have a Gaussian distribution with ferromagnetic mean $\left\langle J_{i j}\right\rangle=J_{0}$. Incorporation of a quadrupolar interaction term and a chemical potential leads to a richer phase diagram with transitions of first and second order. The first order transition may be interpreted as a phase separation, and contrary to what has been argued previously, it persists in the presence of disorder. Finally, the stability of the replica symmetric solution with respect to fluctuations in replica space is analysed, and the transition lines are obtained both analytically and numerically.
\end{abstract}

*E-mail: georg@spht.saclay.cea.fr 


\section{INTRODUCTION}

The much studied SK model of spin glasses may be generalised in different ways. The standard spin glass model, given by

$$
H=-\sum_{<i j>} J_{i j} s_{i} s_{j} \quad \text { with } \quad s_{i}= \pm 1
$$

may be extended for instance by allowing values $s=0, \pm 1, \pm 2, \ldots, \pm S$ for the spin variables. It is then possible to consider higher order interactions such as $K \sum_{i j} s_{i}^{2} s_{j}^{2}$, or a chemical potential such as $\Delta \sum_{i} s_{i}^{2}$. Such generalisations can be regarded as extensions of the BlumeEmery-Griffiths model (BEG) [4]. Indeed the BEG model allows $s=0, \pm 1$ and takes into account the aforementioned higher order interaction and chemical potential. However, in the standard formulation, the bilinear couplings are neither frustrated nor disordered: they are ferromagnetic. The $s=0$ degree of freedom has been used to model a diluted lattice gas and leads to a first order transition separating magnetic and non-magnetic phases. Thus our generalisation of the BEG Hamiltonian is a good way to study the influence of disorder and frustration on first order transitions.

The BEG model has been studied in various contexts and a mean field approximation has been given by Blume, Emery and Griffiths [4]. They introduced the model in order to study multicritical phenomena associated with physical systems such as binary mixtures. For an overview, see the review article of Lawrie and Sarbach [18]. To improve the mean field results and to cope with the finite dimensionality of real physical systems, several different techniques have been applied to the BEG model, e.g., Tucker [24] applied the exponential operator technique of Honmura and Kaneyoshi [16] to the isotropic BEG, and Fittipaldi et al. 12 applied it to the anisotropic BEG model. In addition the model has been treated in the cluster approximation by Tucker 25] and in the local mean field approximation by Maritan et al. [5].

Several disordered BEG models have been studied. Berker et al. [11 looked at a bond disordered BEG model and Ez-Zahraouy [10] looked at a bond diluted BEG model. To our 
knowledge no studies of the case of quenched disorder with frustration have been carried out for the BEG model, though Arenzon et al. [1] have considered a frustrated lattice gas model similar to the BEG model. The closest model to a generalised BEG studied in the literature was first analysed by Ghatak and Sherrington [13]. In their model, they considered $s=0, \pm 1$, and the influence of a chemical potential, but they had no quadrupolar interaction and the ferromagnetic mean $J_{0}$ of their bilinear coupling $J_{i j}$ was assumed to vanish. The interesting point we wish to stress here is that the first order transition of the BEG model, which can be interpreted as a phase separation transition, persists in the GS generalisation. This is in contrast to the findings of Berker et al. which will be discussed below.

In the present paper we generalise the standard SK spin glass Hamiltonian to incorporate the chemical potential, the quadrupolar interaction, randomly distributed bilinear couplings with a non-zero ferromagnetic mean, and other integer values for the spin variables. We restrict our study to the $s=0, \pm 1$ case, which should cover in a qualitative way the general integer $S$-spin models [17]. Furthermore we do not study the effect of disorder in the quadrupolar couplings; such effects have been considered in other models by Snowman et al. [20]. The paper is organised as follows. In section 2 we introduce the model. In section 3 the

free energy is derived in the replica symmetric approximation and in section 4 we give the model's phase diagram. In section 5 we discuss our results in the light of recent arguments of Berker et al. concerning the influence of quenched disorder on phase transitions of first order. In section 6 we analyse the stability of the replica symmetric solution and derive the lines of instability. Conclusions are drawn in the final section.

\section{THE MODEL}

We consider the model described by the BEG-SK Hamiltonian, where the spin variables are allowed to assume the values $s_{i}=0, \pm 1$

$$
H=-\frac{1}{2} \sum_{<i j>} J_{i j} s_{i} s_{j}+\Delta \sum_{i} s_{i}^{2}-\frac{1}{2} K \sum_{<i j>} s_{i}^{2} s_{j}^{2}-h \sum_{i} s_{i}
$$


This model will be treated in the mean field approximation, i.e., in the infinite range limit. For the sake of simplicity we assume, as in the SK model, bilinear couplings with a Gaussian distribution about a non-zero mean $\left\langle J_{i j}\right\rangle=J_{0}>0$ allowing for ferromagnetic ordering. To avoid additional complexity of the model we consider only the case of positive quadrupolar coupling, $K>0$, and a ferromagnetic mean $J_{0}$ of the bilinear couplings. The chemical potential $\Delta$ is allowed to assume positive as well as negative values.

The couplings must be rescaled for the present case of infinite ranged couplings in order to give a sensible free energy in the thermodynamic limit: $J \rightarrow J / \sqrt{N}, J_{0} \rightarrow J_{0} / N$ and $K \rightarrow K / N$, respectively.

$$
p\left(J_{i j}\right)=\sqrt{\frac{N}{2 \pi J^{2}}} e^{\frac{N}{2 J^{2}}\left(J_{i j}-J_{0} / N\right)^{2}} .
$$

We consider now some special cases, which have already been treated in the literature. When $J=0$ and $J_{0}>0$, we recover the non-frustrated and non-disordered standard BEG model, which describes a binary mixture (e.g., $\mathrm{He}^{3}$ and $\mathrm{He}^{4}$ ). When $J_{0}=0$ and $K=0$, we recover the Gathak and Sherrington model. In order to cope with the problem of averaging over quenched disorder, it is necessary to average the free energy over the bond distribution

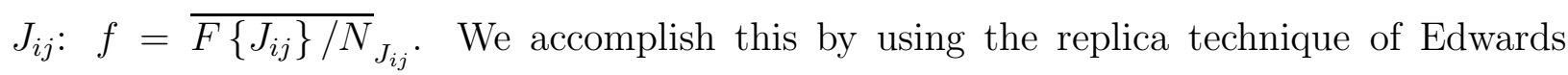
and Anderson [9]. This technique relies on the identity $\ln [Z]=\lim _{n \rightarrow 0} \frac{1}{n}\left(Z^{n}-1\right) ; Z^{n}$ is interpreted as the partition function of a $n$-fold replicated system $s_{i} \rightarrow s_{i}^{\alpha}, \quad \alpha=1, \ldots, n$. The average free energy may be computed using the prescription:

$$
\beta f=-\lim _{n \rightarrow 0} \frac{1}{n}\left(\overline{Z^{n}}-1\right)
$$

We follow this standard procedure to average the logarithm of the partition function. The average of the $n$-fold replicated partition function over the disorder gives

$$
\begin{aligned}
& \left.\overline{Z^{n}}=\operatorname{tr} s_{i}^{\alpha}\right\}=0, \pm 1, \ldots, \pm S \\
& \exp \left\{\frac{\beta^{2} J^{2}}{4 N} \sum_{\alpha \neq \beta} \sum_{i j} s_{i}^{\alpha} s_{i}^{\beta} s_{j}^{\alpha} s_{j}^{\beta}+\left(\frac{\beta^{2} J^{2}}{4 N}+\frac{\beta K}{2 N}\right) \sum_{\alpha} \sum_{i j}\left(s_{i}^{\alpha}\right)^{2}\left(s_{j}^{\alpha}\right)^{2}\right. \\
& \left.+\frac{\beta J_{0}}{2 N} \sum_{\alpha} \sum_{i j} s_{i}^{\alpha} s_{j}^{\alpha}-\beta \Delta \sum_{\alpha} \sum_{i}\left(s_{i}^{\alpha}\right)^{2}+\beta h \sum_{\alpha} \sum_{i} s_{i}^{\alpha}\right\}
\end{aligned}
$$


The Hubbard-Stratonowich transformation $e^{\frac{1}{2} \lambda\left(\sum_{\alpha} s^{\alpha}\right)^{2}}=\int \mathcal{D} t e^{t \sqrt{\lambda} \sum_{\alpha} s^{\alpha}}$ with the convention $\mathcal{D} t=\frac{e^{-\frac{1}{2} t^{2}}}{\sqrt{2 \pi}} \mathrm{d} t$ gives for the free energy, within the framework of the replica method at the saddle point,

$$
\beta f_{n}=\frac{1}{2} \beta J_{0} \sum_{\alpha} m_{\alpha}^{2}+\frac{1}{4} \beta^{2} J^{2} \sum_{\alpha} z_{\alpha}^{2}+\frac{1}{2} \beta K \sum_{\alpha} z_{\alpha}^{2}+\frac{1}{4} \beta^{2} J^{2} \sum_{\alpha \neq \beta} q_{\alpha \beta}^{2}-\ln \left[Z_{\mathrm{eff}}\right],
$$

where the effective Hamiltonian and its partition function is given by

$$
\begin{aligned}
H_{\mathrm{eff}} & =\sum_{\alpha}\left[-\beta \Delta+\beta K z_{\alpha}+\frac{1}{2} \beta^{2} J^{2} z_{\alpha}\right] s_{\alpha}^{2}+\sum_{\alpha}\left[\beta J_{0} m_{\alpha}+\beta h\right] s_{\alpha}+\frac{1}{2} \beta^{2} J^{2} \sum_{\alpha \neq \beta} q_{\alpha \beta} s_{\alpha} s_{\beta} \\
Z_{\mathrm{eff}} & =\operatorname{tr}{ }_{s_{\alpha}=0, \pm 1, \ldots, \pm S} \exp \left\{H_{\mathrm{eff}}\left[s_{\alpha}\right]\right\}
\end{aligned}
$$

The quantities introduced by these transformations acquire the meaning of order parameters:

$$
\begin{aligned}
m_{\alpha} & =<s_{\alpha}> \\
1-x_{\alpha}=z_{\alpha} & =<s_{\alpha}^{2}> \\
q_{\alpha \beta} & =<s_{\alpha} s_{\beta}>,
\end{aligned}
$$

where the average is with respect to the effective Hamiltonian. These results are in fact valid for general integer spin values, but in what follows we restrict ourselves to the case of $S=1$. In order to solve this model it is necessary to make assumptions on the order parameter matrix $q_{\alpha \beta}$ and to propose an Ansatz.

\section{FREE ENERGY OF THE MODEL IN RS}

We limit ourselves to the simplest Ansatz for the order parameter matrix, i.e., we assume symmetry with respect to permutations of any pair of the replicas: $q_{\alpha \beta}=q, \forall \alpha \neq \beta$. The single indexed quantities are assumed to be independent of the replica index: $m_{\alpha}=m$ and $z_{\alpha}=z, \forall \alpha$. This leads to:

$$
\begin{aligned}
& \beta f=\frac{1}{2} \beta J_{0} m^{2}+\frac{1}{2} \beta K z^{2}+\frac{1}{4} \beta^{2} J^{2} z^{2}-\frac{1}{4} \beta^{2} J^{2} q^{2} \\
& -\int \mathcal{D} y \ln \left[1+2 \cosh \left[\beta J_{0} m+\beta h+\beta J \sqrt{q} y\right] e^{\beta K z-\beta \Delta+\frac{1}{2} \beta^{2} J^{2} z-\frac{1}{2} \beta^{2} J^{2} q}\right] .
\end{aligned}
$$


This equation reduces to the one analysed by Ghatak and Sherrington [13, Lage and de Almeida [17] and by Mottishaw and Sherrington [19] if $J_{0}=0$ and if there is no quadrupolar coupling. In order to simplify the notation hereafter, we define

$$
\phi_{k}(y)=\frac{1}{Z_{\mathrm{eff}}(y)} \operatorname{tr}{ }_{s=0, \pm 1, \ldots, \pm S, y}\left[s^{k} e^{-\beta H_{\mathrm{eff}}(y)}\right] .
$$

The effective Hamiltonian and its partition function are the replica symmetric equivalents of those defined earlier and obtained by one further Hubbard-Stratonowich transformation. The set of functions $\phi_{k}(y)$ reduces for the $S=1$ model to just two functions:

$$
\begin{array}{ll}
\phi_{0}(y)=\frac{2 \cosh \left[\beta\left(J_{0} m+y J \sqrt{q}+h\right)\right]}{e^{\beta \Delta+\frac{1}{2} \beta^{2} J^{2} q-\beta \kappa z}+2 \cosh \left[\beta\left(J_{0} m+y J \sqrt{q}+h\right)\right]} & \text { for } k \text { even, } \\
\phi_{1}(y)=\frac{2 \sinh \left[\beta\left(J_{0} m+y J \sqrt{q}+h\right)\right]}{e^{\beta \Delta+\frac{1}{2} \beta^{2} J^{2} q-\beta \kappa z}+2 \cosh \left[\beta\left(J_{0} m+y J \sqrt{q}+h\right)\right]} & \text { for } k \text { odd }
\end{array}
$$

Introducing an effective temperature-dependent quadrupolar coupling $\kappa=K+\frac{1}{2} \beta J^{2}$, the mean field saddle point equations in the replica symmetric approximation are:

$$
\begin{aligned}
& \frac{\partial f}{\partial m}=J_{0} m-J_{0} \int \mathcal{D} y \phi_{1}(y)=0, \\
& \frac{\partial f}{\partial z}=\kappa z-\kappa \int \mathcal{D} y \phi_{0}(y)=0, \\
& \frac{\partial f}{\partial q}=-\frac{1}{2} \beta J^{2} q+\frac{1}{2} \beta J^{2} \int \mathcal{D} y\left[\phi_{1}(y)\right]^{2}=0 .
\end{aligned}
$$

\section{PHASE DIAGRAM}

Mean field phase diagrams are obtained by extremising the free energy with respect to the order parameters. They have been determined for the BEG-SK model by extremising numerically the free energy (6) with respect to the order parameters $z, m$ and $q$ for any temperature $T$ and chemical potential $\Delta$. Following a line of constant chemical potential while varying the temperature — as is shown in figure 5 for two different but fixed values of the chemical potential — will occasionally reveal the onset of ordering. The examples include the appearence of non-vanishing values of the order parameters $m$ or $q$ and discontinous changes of the order parameter $z$ which indicate a phase separation. We infer the 
transition lines by monitoring the magnetisation $m$, the spin glass order parameter $q$, and the concentration $z$ for fixed chemical potential $\Delta$ and varying the temperature $T$. This was performed for a range of different chemical potentials, sufficiently large to exhibit the different phenomenae.

To present the numerical results we will make use of two commonly used phase diagram sections, the section of chemical potential versus temperature $(\Delta-T)$ and the section of concentration versus temperature $(x-T)$. For the sake of completeness we first give the results for the non-disordered BEG model. Since in the BEG model there are two order parameters, the complete phase diagram is 3-dimensional, but we restrict ourselves to the 2-dimensional sections just mentioned. In figure 1 we show the concentration/temperature $(x-T)$ section and in figure 2 the chemical potential/temperature $(\Delta-T)$ section of the phase diagram. Both diagrams are obtained for a small value of the quadrupolar coupling constant $K=0.16$.

Next, we consider a special limit of the BEG-SK model to make contact to the work of Sherrington and Kirkpatrick. We allow the concentration to vary freely and we take the limit $\Delta \rightarrow-\infty$. From this, we recover the Sherrington and Kirkpatrick Hamiltonian [23]: all spins tend to be $|s|=1$, i.e., $x \rightarrow 0$. The phase diagram obtained by Sherrington and Kirkpatrick represents a section of the general phase diagram, i.e., the $x-J_{0}-T$ section for a concentration value $x=0$. This diagram exhibits a paramagnetic phase at high temperatures and small ferromagnetic coupling, whereas it exhibits a ferromagnetic phase for larger ferromagnetic coupling and moderate temperatures. Finally at very low temperatures the spin glass phase appears. This behaviour is shown in figure 3. The figure does not take into account the correction to the transition line between ferromagnetic and spin glass phase due to stability requirements [7].

The section of the general phase diagram obtained by Ghatak and Sherrington [13] is the $\Delta-T$-section for $J_{0}=0$, cf. [22]. The transition lines are obtained analytically following the procedure of Lage and de Almeida [17] by examining the stability of the replica symmetric solution; they are given by 


$$
\Delta=\left\{\begin{array}{r}
\frac{J}{2}+T \ln \left[2\left(\frac{J}{T}-1\right)\right] \quad: \quad T>\frac{1}{3} \\
\frac{J^{2}}{4 T}\left(1 \pm \sqrt{1-\frac{8 T^{2}}{J^{2}}}\right)+T \ln \left[2\left(\frac{1}{\frac{1}{2}\left(1 \pm \sqrt{1-\frac{8 T^{2}}{J^{2}}}\right)}-1\right)\right]: \quad T \leq \frac{1}{3}
\end{array} .\right.
$$

These transition lines are shown in figure 1 . The lower two lines belong respectively to the two choices of sign in the second equation.

Finally we are ready to present the complete phase diagram for BEG-SK model. Figure 5 shows the $x-T$ section and figure 6 the $\Delta-T$ section of the phase diagram for one choice of the coupling parameters: $J_{0}=2.0, J=1.0$ and $K=0.16$. In order to relate this to previous work and to present the complete phase diagram, the $x-T$ and the $\Delta-T$ sections have been calculated for different values of the ferromagnetic coupling (scaled by the variance of the distribution of the couplings) and the results are shown in figures 7 and 8, respectively. Figure 7 shows in the $x-J_{0} / J-T$ plane our numerical results (represented by diamonds) and the results of the SK model (represented by crosses) at a value $x=0$ of the concentration. The line of tricritical temperatures which will be given in formula (23) is shown with a broken line and the surface gives the second order phase transition. In figure 8 the $\Delta-J_{0} / J_{-}$ $T$ section is given and the square symbols indicate the numerical results, which cover the first as well as the second order phase transition. The results from Ghatak and Sherrington 113 are reproduced for the value of the ferromagnetic coupling $J_{0}=0$, i.e., the face of the cube. For details on the stability we refer the reader to section $\mathrm{VI}$ and to figure 11. The first order transitions terminate at the line of tricritical potential given by formula (24), which is the thick line from the bottom left to the top right. The crosses represent the first order transition lines. The second order transition above $T_{\text {tri }}$, given by formula (21) and obtained by the stability analysis presented in Section 6 are represented by squares. The stability lines below $T_{\text {tri }}$ are not given.

We now discuss the numerical results in the light of recent work on disordered BEG-type models which are not frustrated. After we will address the problem of the stability of the replica symmetric solution. 


\section{BEHAVIOR OF THE FIRST ORDER TRANSITION}

Lets look at the influence of disorder on the first order transition, which can — following Blume, Emery and Griffiths — be interpreted as a phase separation. This first order transition represents an example of a transition which breaks a global symmetry. The Hamiltonian is invariant under inversion of all spins; in the ordered phase this symmetry is spontaneously broken, whereas in the paramagnetic phase the symmetry is preserved.

We first consider the non-disordered BEG model in the $x-T$ representation, where the appearance of the phase separation is most visible. In figure 1 the phase diagram is shown for a weak quadrupolar interaction. In figure 9 the same is seen for a rather strong quadrupolar coupling $K=2.88$. The point is that the quadrupolar coupling enhances the phase separation. In the limit of $K>>1$, the model tends to behave as the Griffiths model [14. We thus wish to emphasise the importance of the presence of a quadrupolar interaction. In the original model of Blume, Emery, and Griffiths, even the $K=0$ case exhibits a phase separation. It is due to the bulk interaction of the $s=0$ species $\left(\mathrm{He}^{3}\right)$ and the $s= \pm 1$ species $\left(\mathrm{He}^{4}\right)$, the latter allowing an additional degree of freedom (ferromagnetic phase). At low temperature the ferromagnetic phase is the favoured phase for the $s= \pm 1$ species and phase separation occurs in order to permit ordering in a $\mathrm{He}^{4}$ rich phase. Letting $K \neq 0$ allows for an inter-isotopic interaction; in fact $K$ represents a combination of inter-isotopic interactions, $K=K_{33}+K_{44}-2 K_{34}$, which are assumed to be positive. It is remarkable that for a range of values of $K$ the phase diagram exhibits a triple point together with the critical and tricritical point. This appears in the original BEG model and in the present model, as can be seen from figure 10. The tricritical point is given in the diagrams by $A$, the triple point by $B$, and the critical point by $C$; these special points are also marked in figure 9 .

Introducing disorder in the BEG model may affect the phase separation. Figure 5 shows

the phase diagram obtained by a numerical extremisation of the free energy. The phase separation persists independently of the presence of disorder. The average over the Gaussian disorder has introduced an effective quadrupolar coupling $\kappa$, which — as we have seen before 
in the non-disordered case - enhances the phase separation. Furthermore this effective interaction is also temperature dependent:

$$
\kappa=K+\frac{1}{2} \beta J^{2} .
$$

This promotes the phase separation at low temperatures as can be seen by comparing with figure 9 (for the low temperature regime) and figure 1 (for the high temperature regime) in the non-disordered case. Even if there is an effect due to the presence of disorder, which might suppress or change the first order transition, this effect is apparently compensated by the additional term contributing to the effective quadrupolar coupling.

This result complements those of Berker et al. [2], [3] and [11]. They conjecture, in dimension $d \geq 3$, that the disorder lowers the tricritical temperature; then that part of the transition line enclosed between the former and the actual tricritical temperature should become second order. Also, they claim that all of the first order line is replaced by a second order one if the disorder is sufficiently strong. A few comments are in order:

(i) The analysis of Berker et al. relies on the real space renormalisation group approach for an initially positive distribution of the couplings. This distribution remains positive and consequently cannot take into account the effect of frustration. Nevertheless, we do not think that the frustration is responsible for the persistence of the phase separation for the following reason: even when $J$ is small, so that there is disorder but essentially no frustration, the phase separation persists (see the phase diagrams). Monte Carlo studies by Diep et al. [8] indicate this persistence and hence ensure that the phase separation is not an artefact of the mean field approach.

(ii) The mean field approximation is equivalent to a model in infinite dimensions, perhaps rendering the comparison of our results with the predictions of Berker et al. invalid. Nevertheless, it should give an indication of whether the first order transition persists or not. Furthermore one could argue that in infinite dimensions, an infinitely strong disorder is required to suppress the first order transition. However figure 由 indicates that this model reduces to that discussed by Ghatak and Sherrington 13 with 
a vanishing ferromagnetic coupling, showing again the persistence of the first order transition.

(iii) The assumption of replica symmetry is not justified. However, based on the stability analysis, we have found that the first order transition persists; it seems rather improbable that it be suppressed by doing the full replica symmetry breaking scheme.

To summarise, Berker et al. claim that their results are generic, but our mean field treatment shows that the phase separation is not affected by the introduction of disorder. In particular, the tricritical temperature is not modified: $T / J=1 / 3$, if $J>J_{0}$, using the same scaling as Berker et al. Note that their analysis does not reveal the effective quadrupolar interaction, which plays a subtle role. On the one hand, following Berker et al. and their general arguments [2,3], the disorder should change the first order transition to a second order one; on the other hand, the effective quadrupolar interaction enhances the phase separation, so stabilises the first order transition. As we have seen above, the effective quadrupolar interaction more than compensates the first effect so that the first order transition persists. Furthermore, since for a small amount of disorder the frustration effects are negligible, the models considered are comparable and our results provide a counterexample to their claim.

\section{TRANSITION LINES FROM A STABILITY ANALYSIS}

The assumption of replica symmetry allows for a simple answer for the BEG-SK model. However, as for the SK model, it leads to an unstable solution and the breaking of the permutation symmetry of the replicas is required. We expect the Parisi breaking scheme to apply here and to remove the instabilities in our model. Since under replica symmetry breaking the findings of the analysis assuming replica symmetry are in general confirmed, i.e., a spin glass phase persists, we won't study finite nor infinite step step replica symmetry breaking. In this section we restrict ourselves to the replica symmetric solution and we derive the lines of instability to locate the phase transitions lines given in section IV — even 
though the location of these lines may differ in a more advanced treatment. The stability of the replica symmetric solution of the SK model in its integer spin generalisation was examined by Lage and de Almeida [17] and in greater detail by Mottishaw and Sherrington [19]. These works limit their analysis to the disordered integer $\mathrm{S}$ spin glass model without a non-zero ferromagnetic mean and without quadrupolar coupling.

The present stability analysis follows closely the moethods of de Almeida and Thouless [7] and subsequent works. To examine the stability of the solution it has to be made sure that the solution extremises the free energy. The quadratic form $\Gamma$ describing the deviation of the solution from its stationary value should be positive definite.

$$
\begin{aligned}
\beta f & =\beta f(m, z, q)-\frac{1}{2} \Gamma+\mathcal{O}\left(\delta^{3}\right) \quad \text { and } \\
\Gamma & =\sum_{\alpha \beta} G_{\alpha \beta}^{\epsilon \epsilon} \epsilon_{\alpha} \epsilon_{\beta}+\sum_{\alpha \beta} G_{\alpha \beta}^{\rho \rho} \rho_{\alpha} \rho_{\beta}+\sum_{(\alpha \beta)(\gamma \delta)} G_{(\alpha \beta)(\gamma \delta)}^{\eta \eta} \eta_{\alpha \beta} \eta_{\gamma \delta} \\
& +2 \sum_{\alpha \beta} G_{\alpha \beta}^{\epsilon \rho} \epsilon_{\alpha} \rho_{\beta}+2 \sum_{(\alpha \beta) \gamma} G_{(\alpha \beta) \gamma}^{\eta \epsilon} \eta_{\alpha \beta} \epsilon_{\gamma}+2 \sum_{(\alpha \beta) \gamma} G_{(\alpha \beta) \gamma}^{\eta \rho} \eta_{\alpha \beta} \rho_{\gamma} .
\end{aligned}
$$

The matrix $G$ associated with this form is the Hessian $\mathcal{H}$. The eigenvalues of the Hessian should be non-negative to make sure that the solution is stable. Due to the same symmetry arguments used by de Almeida and Thouless [7], we discover three families of eigenvalues which by convention are called: longitudinal, longitudinal anomalous and replicon. But in the present case the stability analysis requires the diagonalisation of a Hessian $\mathcal{H}$ which is built out of three blocks. As a consequence there are now three distinct eigenvalues in each family. The longitudinal eigenvectors are the fully symmetric ones with respect to permutations of the replica indices and are of the form:

$$
e_{\mu}^{L}=a \text { for } \mu=1 \ldots n, e_{\nu}^{L}=b \text { for } \nu=1 \ldots n, e_{\alpha \beta}^{L}=c \text { for } \alpha, \beta=1 \ldots \frac{n}{2}(n-1)
$$

They give rise to eigenvalues which are the solution to the cubic characteristic equation. The full formula for the eigenvalues is too cumbersome to be displayed here, they will be discussed in the paramagnetic phase below (the full expressions are given in appendix A). The longitudinal anomalous eigenvectors are generated by one distinct replica index $\theta$ and look like: 


$$
\begin{aligned}
& e_{\mu}^{L A}=a \text { for } \mu=1 \ldots n \text { and } \mu \neq \theta, e_{\mu}^{L A}=g \text { for } \mu=\theta \\
& e_{\nu}^{L A}=b \text { for } \nu=1 \ldots n \text { and } \mu \neq \theta, e_{\nu}^{L A}=c \text { for } \nu=\theta \\
& e_{\alpha \beta}^{L A}=d \text { for } \alpha, \beta=1 \ldots \frac{n}{2}(n-1) \text { and } \alpha, \beta \neq \theta, e_{\alpha \beta}^{L A}=e \text { for } \alpha, \beta=\theta .
\end{aligned}
$$

Again the eigenvalues are given in appendix A. The replicon eigenvectors are generated by two distinct replica indices $\omega$ and $\theta$ and they are of the following form:

$$
\begin{aligned}
& e_{\mu}^{R}=a \text { for } \mu=1 \ldots n \text { and } \mu \neq \theta, \omega, e_{\mu}^{R}=g \text { for } \mu=\theta, \omega, \\
& e_{\nu}^{R}=b \text { for } \nu=1 \ldots n \text { and } \mu \neq \theta, \omega, e_{\nu}^{R}=c \text { for } \nu=\theta, \omega, \\
& e_{\alpha \beta}^{R}=d \text { for } \alpha, \beta=1 \ldots \frac{n}{2}(n-1) \text { and } \alpha, \beta \neq \theta \text { and } \alpha, \beta \neq \omega, \\
& e_{\alpha \beta}^{R}=e \text { for } \alpha, \beta=\theta \text { or } \alpha, \beta=\omega, e_{\alpha \beta}^{R}=f \text { for }(\alpha \beta)=(\theta \omega) .
\end{aligned}
$$

The eigenvalue is given by the solution of the characteristic equation, but as this equation is explicit, so the eigenvalue is obtained immediately:

$$
\lambda_{R}=P-2 Q+R,
$$

where $P, Q$ and $R$ are appropriate correlation functions given in the appendix. Adding the number of eigenvalues obtained in the three symmetry families gives the expected number $3+(3 n-3)+\frac{n}{2}(n-3)=\frac{n}{2}(n+3)$, i.e., the dimension of $\mathcal{H}$. The so called longitudinal eigenvalues $\lambda_{L}$ and $\lambda_{L A}$ coincide in the $n \rightarrow 0$-limit as in the work of de Almeida and Thouless [7] and Lage and de Almeida [17]. The replica symmetric fluctuations of the one-indexed quantities $\left(z_{\alpha}\right.$ and $\left.m_{\alpha}\right)$ and the two-indexed quantity $q_{\alpha \beta}$ are described respectively by the longitudinal eigenvalues, $\lambda_{L A}$ and $\lambda_{L}$. The third eigenvalue $\lambda_{R}$ is distinct from the other two eigenvalues and is related to the fluctuations which break the replica symmetry of the two indexed quantity $q_{\alpha \beta}$. In order to investigate the stability of the replica symmetric solution, for example in the ferromagnetic phase, the eigenvalues $\lambda_{L}, \lambda_{L A}$ and $\lambda_{R}$ have to be calculated in this phase. In the appendix the general expressions for the different eigenvalues and the correlation functions appearing therein are given. They must be evaluated under the appropriate approximation, i.e., in the present case, under the replica symmetric 
approximation. In order for a phase to be stable the eigenvalues in this phase have to be non-negative. We now investigate the stability of the paramagnetic phase.

\section{The paramagnetic phase}

Consider the paramagnetic phase defined by the vanishing of all magnetic order parameters: $q=0, m=0$. This considerably simplifies the expressions for the eigenvalues. All off-diagonal elements of the Hessian or combinations appearing in the characteristic

equations vanish. In order to simplify the notation further, we consider directly the combinations appearing in the definitions of the different eigenvalues. The longitudinal eigenvalues are given by the solution of:

$$
\lambda_{L}^{3}+a \lambda_{L}^{2}+b \lambda_{L}+c=\left(\lambda-\lambda_{1}\right)\left(\lambda-\lambda_{2}\right)\left(\lambda-\lambda_{3}\right)=0
$$

The coefficients for a cubic equation are given implicitly by the solutions to that equation:

$$
\begin{aligned}
-a & =[U-V]+[A-B]+[P-4 Q+3 R]=\lambda_{1}+\lambda_{2}+\lambda_{3} \\
b & =[U-V][P-4 Q+3 R]+[A-B][P-4 Q+3 R]+[U-V][A-B]=\lambda_{1} \lambda_{2}+\lambda_{2} \lambda_{3}+\lambda_{1} \lambda_{3} \\
c & =-[U-V][A-B][P-4 Q+3 R]=-\lambda_{1} \lambda_{2} \lambda_{3},
\end{aligned}
$$

which may be solved immediately to give

$$
\begin{aligned}
& \lambda_{1}^{L}=[A-B] \\
& \lambda_{2}^{L}=[U-V] \\
& \lambda_{3}^{L}=[P-4 Q+3 R]=P .
\end{aligned}
$$

The longitudinal anomalous eigenvalues are given by the same expressions. The replicon eigenvalue is given by:

$$
\lambda_{R}=P-2 Q+R=P,
$$

and coincides in the paramagnetic phase with the third longitudinal eigenvalue. In order for the paramagnetic phase to be stable, the eigenvalues in this phase must be non-negative. 
The border line of stability is given by $\lambda_{L, L A, R}=0$, which defines seven lines; but due to the collapsing eigenvalues of different families there are only three different stability lines. In the replica symmetric approximation, these read:

$$
\begin{aligned}
A-B & =\beta J_{0}\left[1-\beta J_{0} z\right]=0 \\
U-V & =\beta \kappa\left[1-\beta \kappa\left(z-z^{2}\right)\right]=0 \\
P-4 Q+3 R=P-2 Q+R & =\beta^{2} J^{2}\left[1-\beta^{2} J^{2} z^{2}\right]=0 .
\end{aligned}
$$

The stability limits are given by the following explicit formulae, first in the plane of concentration $x$ versus temperature $T$ :

$$
\begin{aligned}
\frac{T(x)}{J_{0}} & =1-x \\
\frac{T(x)}{J} & =1-x \\
\frac{1}{\beta K+\frac{1}{2} \beta^{2} J^{2}} & =x(1-x) .
\end{aligned}
$$

The paramagnetic phase becomes unstable whenever the temperature is lowered below the greater of the two bilinear couplings $J$ and $J_{0}$. This behaviour is identical to that observed by Sherrington and Kirkpatrick. The conditions of stability can be rewritten in terms of the chemical potential $\Delta$ and temperature $T$. The stability lines are obtained by using the fixed point equation (8) determinating the concentration, and represent a line in the $\Delta-T$-section of the phase diagram.

$\Delta_{\mathrm{RS}}(T)=\left\{\begin{array}{rl}\left(K+\frac{1}{2} \beta J^{2}\right) \frac{T}{J}+T \ln \left[2\left(\frac{J}{T}-1\right)\right] \quad: \quad T>T_{\text {tri }} \\ \left(K+\frac{1}{2} \beta J^{2}\right) \frac{1}{2}\left(1 \pm \sqrt{1-\frac{8}{2 \beta K+\beta^{2} J^{2}}}\right)+T \ln \left[2\left(\frac{1}{\frac{1}{2}\left(1 \pm \sqrt{1-\frac{8}{2 \beta K+\beta^{2} J^{2}}}\right)}-1\right)\right]: \quad T \leq T_{\mathrm{tri}}\end{array}\right.$.

For one choice of the parameter the complete set of stability lines has been depicted in figure [1]. Furthermore the numerical results covering the first order transition points as well as those of second order are represented by diamond symbols. The following discussion refers to this figure. The two choices of sign in the second equation separate different regions 
of the free energy's behaviour. The branch $(\mu)$ belonging to the positive sign separates the region of free energy with a unique minimum (PM-U) from the region where there is more than one local minima (PM-M). It is in this latter region where first order transitions occur and the phase separation appears. The branch $(\varrho)$ belonging to the negative sign represents regions where the free energy has three local minima, separating the region where the minimal solution is the one with vanishing order parameter from the region where the minimal solution is the one with a nonvanishing order parameter; this line $(\varrho)$ is indicating the onset of phase separation. The concentration $x$ jumps when crossing this line; in fact this line represents in the $\Delta-T$ section of the phase diagram the whole phase separation or coexistence region. As can be seen by comparision with the numerical data, the location of the first order phase transition is predicted correctly only near the tricritical point. It is known that the stability analysis is not an adequate mean to determine first order phase transitions. As in previous works the $\lambda$-line $(\lambda)$ meets the first order transition line at a tricritical point: a second order line changes to a first order line, or, following Griffiths [15], three critical lines meet (see also [18]). For completeness the continuation of the $\lambda$-line below $T_{\text {tri }}$ has been drawn too.

\section{Tricriticality}

The occurrence of a tricritical point in a system with one order parameter is signaled by the vanishing of the fourth derivative of the free energy with respect to the order parameter. In the present problem there are three order parameters and they are have replica indices. The criterion must be modified: the eigenvalues of the matrix of the fourth derivatives have to vanish. But due to the diagonal structure of the Hessian $\mathcal{H}$ in the paramagnetic phase this amounts to computing the fourth derivative with respect to the magnetisation and to search for its zero. This is in fact the classical argument of the Landau theory. The tricritical temperature in the non-disordered case is given for a vanishing quadrupolar coupling by

$$
T_{\text {tri }}=\frac{1}{3} \max \left\{J_{0}, J\right\}
$$


For a non-vanishing quadrupolar coupling the tricritical temperature is given by:

$$
T_{\mathrm{tri}}=\frac{2 K+1}{2 K+3} \max \left\{J_{o}, J\right\} .
$$

The tricritical point present in the non-disordered model (BEG) persists and in terms of the temperature and concentration is given in the crudest approximation by:

$$
x_{\mathrm{tri}}=\frac{2}{3} \quad \text { and } \quad T_{\mathrm{tri}}=\frac{2 K+1}{2 K+3} \max \left\{J_{0}, J\right\} .
$$

The numerical data show that this is a good approximation. It is worth mentioning that the tricritical concentration has kept its value from the non-disordered BEG model. In contrast the tricritical chemical potential has been modified. Resolving the tricritical condition provides a formula in $\Delta-J_{0} / J-T$ space for the tricritical line. In the $\Delta-T$ plane, it is given by:

$$
\Delta_{\text {tri }}\left(J_{0}, J\right)=\left\{\begin{array}{ccc}
\frac{K}{3}+\frac{1}{2} \frac{J^{2}}{J_{0}}+\frac{2}{3} J_{0} \ln [2] & \text { and } & T_{\text {tri }}=\frac{J_{0}}{3} \text { for } J_{0}>J \\
\frac{K}{3}+\frac{1}{2} J+\frac{2}{3} J \ln [2] & \text { and } & T_{\text {tri }}=\frac{J}{3} \text { for } J>J_{0}
\end{array} .\right.
$$

This line, as a function of $J_{0}$, is constant up to $J_{0}=J$, and then tends for stronger ferromagnetic coupling to larger chemical potential. The line of stability for the second order transition and the aforementioned line of tricritical $\Delta$ are displayed together with the numerical data in figure 8. The value for the quadrupolar coupling for this figure is $K=0.16$.

\section{DISCUSSION AND CONCLUSIONS}

We have performed a replica study of the disordered BEG model and have extended previous work to present a picture of the complete phase diagram. The BEG-SK model shows a second order transition, the so-called $\lambda$-line, separating an ordered phase from a paramagnetic one. The ordered phase may be a ferromagnetic or a spin glass phase, depending on the strength of the ferromagnetic coupling $J_{0}$. Furthermore, a transition of first order, present in the non-disordered BEG model, persists, and may be interpreted as 
a phase separation. This extends recent work of Berker et al. on the influence of disorder

on first order transitions. Our mean field study is completed by a stability analysis of the replica symmetric approximation. The complete set of eigenvectors and eigenvalues has been found and analysed in the paramagnetic phase. The replica symmetric solution suffers from instabilities, but the assumption of replica symmetry and the associated results do not exhibit more severe inconsistencies than in the SK model. For the SK model, the instabilities of the replica symmetric solution have been remedied by the infinite step replica symmetry breaking procedure. We expect the same approach to work in this case, but following Mottishaw and Sherrington [19] near the tricritical point, the Parisi Ansatz has to be extended to higher order in $q_{\alpha \beta}$.

\section{ACKNOWLEDGEMENT}

I would like to thank Thomas Garel for suggesting this problem, T. H. Diep, C. de Dominicis, O. Martin, H. Orland for helpful discussions, and A. Sedeki for checking some of the computatations. An Individual Fellowship of the Commission of European Communities under contract number ERBCHBICT941665 is gratefully acknowledged. Furthermore, I would like to express my gratitude to Professors A. Poves and J. Luis Egido for their generous hospitality at the Department of Theoretical Physics of the Universidad Autonoma de Madrid, where part of this work was accomplished. The Division de Physique Théorique is an Unité de Recherche des Universités Paris XI et Paris VI, associée au C.N.R.S.

\section{APPENDIX A: EIGENVECTORS AND EIGENVALUES}

We follow Lage and de Almeida [17] in their stability analysis of the replica symmetric solution. The Hessian of the free energy is given schematically by: 


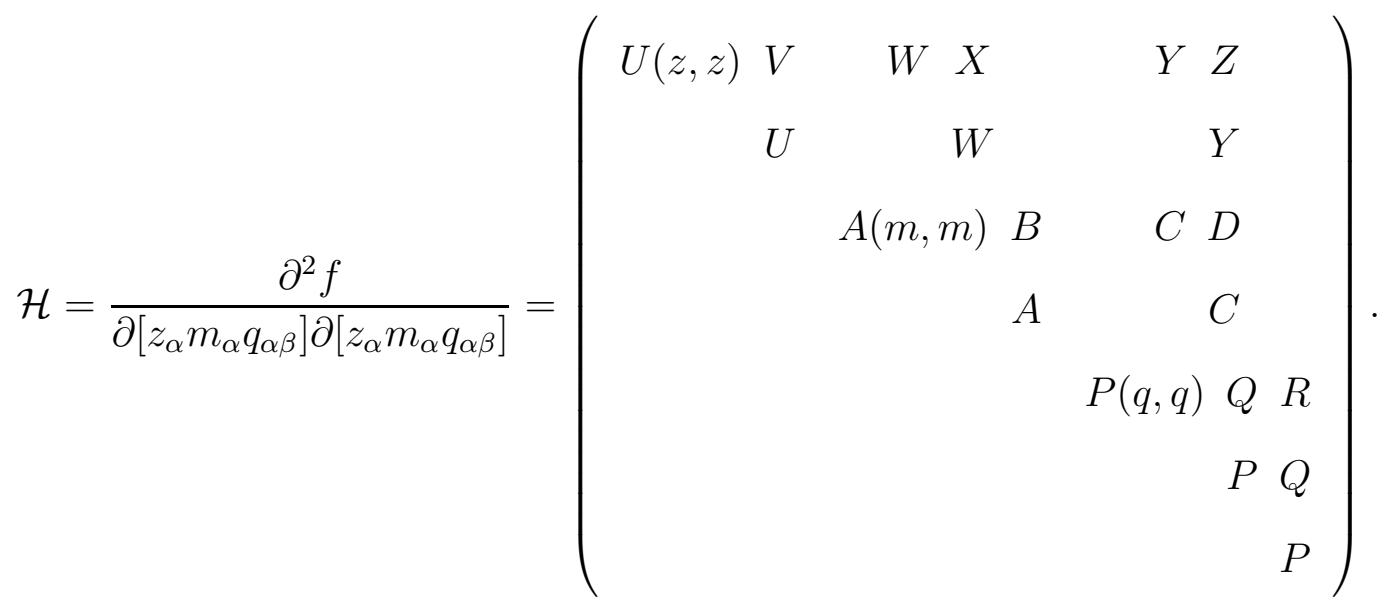

The second equality defines the quantities $U, V, \ldots$ with respect to their position in the Hessian. These quantities are the respective $G_{\epsilon \epsilon}^{\alpha \beta}$, etc ... as given in the main part. As will be seen later they can be expressed in terms of the different multispin correlation functions up to degree 4. The dimension of the Hessian is $n+n+\frac{n}{2}(n-1)=\frac{n}{2}(n+3)$ and equals the number of eigenvalues and eigenvectors to be found. In order to diagonalise the Hessian we construct the eigenvectors using the symmetry arguments exposed by de Almeida and Thouless [7]. We do not construct a orthonormal set of eigenvectors, because the additional constraints destroy the symmetry with respect to replica permutations. Rather we content ourselves with three families of eigenvectors, each orthogonal to another, but not orthogonal within the families.

We start with the eigenvector totally symmetric under permutations of the replicas:

$$
\mathbf{e}_{L}= \begin{cases}a & : \quad \mu=1 \ldots n \\ b & : \quad \nu=1 \ldots n \\ c & : \quad \alpha, \beta=1 \ldots \frac{n}{2}(n-1)\end{cases}
$$

According to conventional notation this vector is referred to as belonging to the longitudinal subspace; hence the subscript $L$. This is a subspace of dimension $d=3$, which is easily verified by constructing a orthogonal set of eigenvectors conserving the form prescribed above. The eigenvector equation $\mathcal{H} \mathbf{e}_{L}=\lambda_{L} \mathbf{e}_{L}$ reads

$$
a\left[U+(n-1) V-\lambda_{L}\right]+b[W+(n-1) X]+c\left[Y(n-1)+Z\left[\frac{n}{2}(n-1)-(n-1)\right]\right]=0
$$




$$
\begin{array}{r}
a[W+(n-1) X]+b\left[A+(n-1) B-\lambda_{L}\right]+c\left[C(n-1)+D\left[\frac{n}{2}(n-1)-(n-1)\right]\right]=0 \\
a[2 Y+(n-2) Z]+b[2 C+(n-2) D]+c\left[P+2(n-2) Q+R\left[\frac{n}{2}(n-1)-2(n-1)-1\right]-\lambda_{L}\right]=0
\end{array}
$$

The longitudinal eigenvector equation gives rise to a cubic characteristic equation for the eigenvalue $\lambda_{L}$ :

$$
\left(\lambda^{L}\right)^{3}+a\left(\lambda^{L}\right)^{2}+b \lambda^{L}+c=0 .
$$

We give its coefficients in the $n=0$ limit:

$$
\begin{aligned}
a= & -[U-V]-[A-B]-[P-4 Q+3 R]=-\lambda_{1}^{L}-\lambda_{2}^{L}-\lambda_{3}^{L} \\
b= & -[W-X]^{2}+[U-V][P-4 Q+3 R]+2[Y-Z]^{2} \\
& +[A-B][P-4 Q+3 R]+[U-V][A-B]+2[C-D]^{2}=\lambda_{1}^{L} \lambda_{2}^{L}+\lambda_{2}^{L} \lambda_{3}^{L}+\lambda_{1}^{L} \lambda_{3}^{L} \\
c= & -[U-V][A-B][P-4 Q+3 R]-2[U-V][C-D]^{2} \\
& +[W-X]^{2}[P-4 Q+3 R]+4[W-X][Y-Z][C-D]-2[Y-Z]^{2}[A-B]=-\lambda_{1}^{L} \lambda_{2}^{L} \lambda_{3}^{L} .
\end{aligned}
$$

Define the following quantities:

$$
\begin{aligned}
\gamma & =-\frac{a^{2}}{3}+b \\
\varrho & =2\left(\frac{a}{3}\right)^{3}-\frac{1}{3} a b+c \\
\Gamma & =\left(\frac{\gamma}{3}\right)^{3}+\left(\frac{\varrho}{2}\right)^{2} \\
\sigma^{+} & =\left(-\frac{\varrho}{2}+\sqrt{\Gamma}\right)^{\frac{1}{3}} \\
\sigma^{-} & =\left(-\frac{\varrho}{2}-\sqrt{\Gamma}\right)^{\frac{1}{3}},
\end{aligned}
$$

where only the real cubic roots are used. This allows us to write the three solutions as:

$$
\begin{aligned}
& \lambda_{L 1}=\sigma^{+}+\sigma^{-} \\
& \lambda_{L 2}=-\frac{1}{2}\left(\sigma^{+}+\sigma^{-}\right)+\frac{i}{2} \sqrt{3}\left(\sigma^{+}-\sigma^{-}\right) \\
& \lambda_{L 3}=-\frac{1}{2}\left(\sigma^{+}+\sigma^{-}\right)-\frac{i}{2} \sqrt{3}\left(\sigma^{+}-\sigma^{-}\right) .
\end{aligned}
$$

The value of $\Gamma$ tells us whether the solutions are degenerate or not. 


$$
\Gamma\left\{\begin{array}{lll}
<0 & : & \text { distinct real solutions } \\
=0 & : & \text { degenerate real solutions } \\
>0 & : & \text { complex solutions }
\end{array}\right.
$$

As has been recognized by Lage and de Almeida [17], occasionally $\Gamma$ is positive and so the eigenvalues become complex. This has been verified numerically by da Costa et al. [6].

The next eigenvectors, which will be called longitudinal anomalous, are constructed by breaking the symmetry of the longitudinal vector with respect to one replica, given by the distinct index $\theta$.

$$
\mathbf{e}_{L A}= \begin{cases}a & : \quad \mu=1 \ldots n, \mu \neq \theta \\ g & : \quad \mu=\theta \\ b & : \quad \nu=1 \ldots n, \mu \neq \theta \\ c & : \quad \nu=\theta \\ d & : \quad \alpha, \beta=1 \ldots \frac{n}{2}(n-1), \alpha, \beta \neq \theta \\ e & : \quad \alpha, \beta=\theta\end{cases}
$$

Consider the orthogonality condition for the first eigenvector, if $a=g$ or $b=c$. This condition results in trivial eigenvectors. In order to obtain non-trivial eigenvectors, the symmetry in both $n$-blocks has to be broken, giving $k n$ eigenvectors of the second family, where $k$ is the number of different choices of the parameters conserving the prescribed form. Orthogonality of the second family to the first family of eigenvectors requires:

$$
g=(1-n) a \quad c=(1-n) b \quad e=\left(1-\frac{n}{2}\right) d .
$$

Therefore $k=3$ and this choice gives rise to $3 n$ eigenvectors including the previous one. Writing down the characteristic equation for the longitudinal anomalous eigenvalues $\lambda_{L A}$ gives:

$$
\begin{array}{r}
a\left[U-V-\lambda_{L A}\right]+b[W-X]+d\left(\frac{n}{2}-1\right)[Y-Z]=0 \\
a[W-X]+b\left[A-B-\lambda_{L A}\right]+d\left(\frac{n}{2}-1\right)[C-D]=0 \\
2 a[Y-Z]+2 b[C-D]+d\left(\frac{n}{2}-1\right)\left[P+(n-4) Q+R(3-n)-\lambda_{L A}\right]=0
\end{array}
$$


The longitudinal anomalous eigenvector equation also gives rise to a cubic characteristic equation for the eigenvalue $\lambda_{L}$ :

$$
\left(\lambda^{L A}\right)^{3}+a\left(\lambda^{L A}\right)^{2}+b \lambda^{L A}+c=0 .
$$

We quote its coefficients in the $n=0$ limit:

$$
\begin{aligned}
a= & -U+V-A+B-P+4 Q-3 R \\
b= & (W-X)^{2}-(U-V)(P-4 Q+3 R)+(2 Y-2 Z)(-Y+Z) \\
& -(A-B)(P-4 Q+3 R)-(U-V)(A-B)+(-C+D)(2 C-2 D) \\
c= & (U-V)(A-B)(P-4 Q+3 R)-(U-V)(-C+D)(2 C-2 D) \\
& -(W-X)^{2}(P-4 Q+3 R)+(W-X)(-Y+Z)(2 C-2 D) \\
& +(2 Y-2 Z)(W-X)(-C+D)-(2 Y-2 Z)(-Y+Z)(A-B)
\end{aligned}
$$

Again the solutions may be written as in equation A1, but now with modified coefficients $\gamma, \varrho, \ldots$

The eigenvalues will in general be different in each family, but since the characteristic equations for the longitudinal and the anomalous longitudinal become identical in $n=0$ limit, so do the eigenvalues, and then the longitudinal and longitudinal anomalous families collapse. There are $3 n$ eigenvectors and the three eigenvalues are each $n$-fold degenerate, including the three eigenvalues of the first eigenvector.

Finally, there is the third family, called replicon. Breaking the symmetry with respect to permutations of pairs by distinguishing two indices $\theta$ and $\omega$, we obtain the following form for the eigenvectors: 


$$
\mathbf{e}_{R}= \begin{cases}a & : \quad \mu=1 \ldots n, \mu \neq \theta, \omega \\ g & : \quad \mu=\theta, \omega \\ b & : \quad \nu=1 \ldots n, \mu \neq \theta, \omega \\ c & : \quad \nu=\theta, \omega \\ d \quad: \quad & \alpha, \beta=1 \ldots \frac{n}{2}(n-1), \alpha, \beta \neq \theta \text { and } \alpha, \beta \neq \omega \\ e & : \quad \alpha, \beta=\theta \text { or } \alpha, \beta=\omega \\ f & : \quad(\alpha \beta)=(\theta \omega)\end{cases}
$$

Orthogonality of the third family to the previous two families of eigenvectors requires:

$$
g=a \quad c=b \quad 0=f+(n-4) e+(3-n) d .
$$

The choice of equal non-vanishing entries would give the first family, so the recommended choice is

$$
g=a=0 \quad c=b=0 \quad f=(2-n) e \quad e=\frac{1}{2}(3-n) d .
$$

The characteristic equation is the solution for the replicon eigenvalue itself:

$$
\lambda_{R}=P-2 Q+R
$$

The replicon eigenvalue is independent of $n$ and identical in form to the result obtained by de Almeida and Thouless [7]. This eigenvalue is $\frac{n}{2}(n-3)$-fold degenerate.

\section{The replica symmetric Ansatz}

In order to analyse the stability of the replica symmetric Ansatz, the quantities defined in the Hessian are to be evaluated assuming replica symmetry. This gives:

$$
\begin{aligned}
& A=\beta J_{0}\left[1-\beta J_{0}\left(<s_{\alpha}^{2}>-<s_{\alpha}>^{2}\right)\right]=\beta J_{0}\left[1-\beta J_{0}\left(z-m^{2}\right)\right] \\
& B=\beta^{2} J_{0}^{2}\left[<s_{\alpha}>^{2}-<s_{\alpha} s_{\beta}>\right]=\beta^{2} J_{0}^{2}\left[m^{2}-q\right] \\
& C=\beta^{2} J^{2} \beta J_{0}\left[<s_{\alpha}><s_{\alpha} s_{\beta}>-<s_{\alpha} s_{\beta}^{2}>\right]=\beta^{2} J^{2} \beta J_{0}[m q-r]
\end{aligned}
$$




$$
\begin{aligned}
& D=\beta^{2} J^{2} \beta J_{0}\left[<s_{\gamma}><s_{\alpha} s_{\beta}>-<s_{\alpha} s_{\beta} s_{\gamma}>\right]=\beta^{2} J^{2} \beta J_{0}[m q-u] \\
& P=\beta^{2} J^{2}\left[1-\beta^{2} J^{2}\left(<s_{\alpha}^{2} s_{\beta}^{2}>-<s_{\alpha} s_{\beta}>^{2}\right)\right]=\beta^{2} J^{2}\left[1-\beta^{2} J^{2}\left(s-q^{2}\right)\right] \\
& Q=\beta^{4} J^{4}\left[<s_{\alpha} s_{\beta}>^{2}-<s_{\alpha}^{2} s_{\beta} s_{\gamma}>\right]=\beta^{4} J^{4}\left[q^{2}-v\right] \\
& R=\beta^{4} J^{4}\left[<s_{\alpha} s_{\beta}>^{2}-<s_{\alpha} s_{\beta} s_{\gamma} s_{\delta}>\right]=\beta^{4} J^{4}\left[q^{2}-w\right] \\
& U=\beta \kappa\left[1-\beta \kappa\left(<s_{\alpha}^{2}>-<s_{\alpha}^{2}>^{2}\right)\right]=\beta \kappa\left[1-\beta \kappa\left(z-z^{2}\right)\right] \\
& V=\beta^{2} \kappa^{2}\left[<s_{\alpha}^{2}>^{2}-<s_{\alpha}^{2} s_{\beta}^{2}>\right]=\beta^{2} \kappa^{2}\left[z^{2}-s\right] \\
& W=\beta J_{0} \beta \kappa\left[<s_{\alpha}>\left(<s_{\alpha}^{2}>-1\right)\right]=\beta J_{0} \beta \kappa[m(z-1)] \\
& X=\beta J_{0} \beta \kappa\left[<s_{\alpha}><s_{\alpha}^{2}>-<s_{\alpha} s_{\beta}^{2}>\right]=\beta J_{0} \beta \kappa[m z-r] \\
& Y=\beta^{2} J^{2} \beta \kappa<s_{\alpha} s_{\beta}>\left[<s_{\alpha}^{2}>-1\right]=\beta^{2} J^{2} \beta \kappa q[z-1] \\
& Z=\beta^{2} J^{2} \beta \kappa\left[<s_{\alpha}^{2}><s_{\alpha} s_{\beta}>-<s_{\alpha}^{2} s_{\beta} s_{\gamma}>\right]=\beta^{2} J^{2} \beta \kappa[z q-v] .
\end{aligned}
$$

The second equality in each line results from the assumption of replica symmetry. Additional simplifications are due to the fact that for the $S=1$ spin model there are additional relations, e.g., $S^{2}=S^{4}$, etc. Using the previously defined functions $\phi_{k}(y)$, see equation (7), the replica symmetric correlation functions introduced above may be explicitly written as:

$$
\begin{array}{ll}
m=<s_{\alpha}>=\int \mathcal{D} y \phi_{1}(y) & t=<s_{\alpha}^{3} s_{\beta}>=\int \mathcal{D} y \phi_{1}(y)^{2} \\
z=\left\langle s_{\alpha}^{2}>=\int \mathcal{D} y \phi_{0}(y)\right. & u=<s_{\alpha} s_{\beta} s_{\gamma}>=\int \mathcal{D} y \phi_{1}(y)^{3} \\
q=<s_{\alpha} s_{\beta}>=\int \mathcal{D} y \phi_{1}(y)^{2} & v=<s_{\alpha}^{2} s_{\beta} s_{\gamma}>=\int \mathcal{D} y \phi_{1}(y)^{2} \phi_{0}(y) \\
r=<s_{\alpha} s_{\beta}^{2}>=\int \mathcal{D} y \phi_{1}(y) \phi_{0}(y) & w=<s_{\alpha} s_{\beta} s_{\gamma} s_{\delta}>=\int \mathcal{D} y \phi_{1}(y)^{4} . \\
s=<s_{\alpha}^{2} s_{\beta}^{2}>=\int \mathcal{D} y \phi_{0}(y)^{2} &
\end{array}
$$

\section{Paramagnetic phase}

As is seen from the definitions of the multispin correlation functions (see equations A5 and A6) some of these vanish identically in the paramagnetic phase. Furthermore some of 
the combinations appearing in the calculation of the eigenvalues, e.g. $[W-X]=0$, vanish too. This simplifies significantly the stability analysis in the paramagnetic phase, because the Hessian $\mathcal{H}$ and the matrix of the fourth derivatives become diagonal. 


\section{REFERENCES}

[1] Jeferson J. Arenzon, Mario Nicodemi, and Mauro Sellitto. Equilibrium properties of the Ising frustrated lattice gas. Journal de Physique France, September 1996. to be published.

[2] A. Nihat Berker. Absence of temperature-driven first-order phase transitions in systems with random bonds. J. Appl. Phys., 70(10):5941 - 5945, November 1991.

[3] A. Nihat Berker. Critical behavior induced by quenched disorder. Physica A, 194:72$76,1993$.

[4] M. Blume, V. J. Emery, and R. B. Griffiths. Ising Model for the $\lambda$ Transition and Phase Separation in $\mathrm{He}^{3}-\mathrm{He}^{4}$ Mixtures. Phys. Rev. A, 4(3):1071 - 1077, September 1971.

[5] Carla Buzano, Amos Maritan, and Alessandro Pelizzola. A cluster variation approach to the random-anisotropy Blume-Emery-Griffiths model. Journal of Physics C: Condensed Matter, 6:327 - 338, 1994.

[6] Francisco A. da Costa, Carlos S. O. Yokoi, and Silvio R. A. Salinas. First-order transition in a spin-glass model. J. Phys. A, 27:3365 - 3372, 1994.

[7] J.R.L. de Almeida and D.J. Thouless. Stability of the Sherrington-Kirkpatrick Solution of a spin glass model. J. Phys. A, 11:983-990, June 1978.

[8] T. H. Diep. Private communication. 1997.

[9] S. F. Edwards and P. W. Anderson. Theory of spin glasses. J. Phys. F: Metal Physics, 5:965 - 974, May 1975.

[10] H. Ez-Zahraouy. The Bond Diluted Spin-1 Blume-Emery-Giffiths Model in a Transverse Field. Physica Scripta, 51:310 - 315, 1995.

[11] Alexis Falicov and A. Nihat Berker. Tricritical and critical-endpoint phenomena under random bonds. preprint, 1995. 
[12] I. P. Fittipaldi and T. Kaneyoshi. Re-entrant behaviour of the anisotopic BEG model in the effective-field approximation. Journal of Physics C: Condensed Matter, 1:6513$6522,1989$.

[13] S. K. Ghatak and D. Sherrington. Crystal field effects in a general S Ising spin glass. J. Phys. C: Solid State Physics, 10:3149 - 3156, 1977.

[14] R. B. Griffiths. First-order phase transitions in spin-one Ising systems. Physica, 33:689 - 690, 1967.

[15] Robert B. Griffiths. Thermodynamics near the two-fluid critical mixing point in $\mathrm{He}^{3}-$ $\mathrm{He}^{4}$. Phys. Rev. Lett., 24(13):689 - 690, 1970.

[16] R. Honmura and T. Kaneyoshi. Contribution to the new type of effective-field theory of the Ising model. Journal of Physics C: Condensed Matter, 12:3979 - 3992, 1979.

[17] E. J. S. Lage and J. R. L. de Almeida. Stability conditions of generalised Ising spin glass models. J. Phys. C: Solid State Physics, 15:L1187 - L1193, 1982.

[18] Ian D. Lawrie and Stéphane Sarbach. Theory of Tricritical Points. In C. Domb and J. L. Lebowitz, editors, Phase Transitions and Critical Phenomena, Vol.9, Phase Transitions and Critical Phenomena, chapter 1, pages 1 - 161. Academic Press, London, September 1984.

[19] P. J. Mottishaw and D. Sherrington. Stability of a crystal-field split spin glass. J. Phys. C: Solid State Physics, 18:5201 - 5213, 1985.

[20] Snowman Daniel P. and Susan R. McKay. The effects of frustrated biquadratic interactions on the phase diagrams and criticality of the Blume-Emery-Griffiths model. J. Appl. Phys., 76(10):7117, November 1994.

[21] G. Parisi. Magnetic properties of spin glasses in a new mean field theory. J. Phys. A: Math. Gen., 13:1887 - 1895, 1980. 
[22] Abdelouahab Sedeki. Rapport de stage du DEA. rapport interne, Université de CergyPontoise, Groupe de Physique Statistique, 1994. unpublished.

[23] D. Sherrington and S. Kirkpatrick. Solvable model of a spin glass. Phys. Rev. Lett., 35(26):1792 - 1796, June 1975.

[24] J. W. Tucker. The effective-field theory of the isotropic Blume-Emery-Griffiths model. Journal of Physics C: Condensed Matter, 21:6215 - 6218, 1988.

[25] J. W. Tucker, M. Saber, and L. Peliti. A new technique in the effective field theory of general spin S dilute Ising models. Physica A, 206:497 - 507, 1994. 


\section{FIGURES}

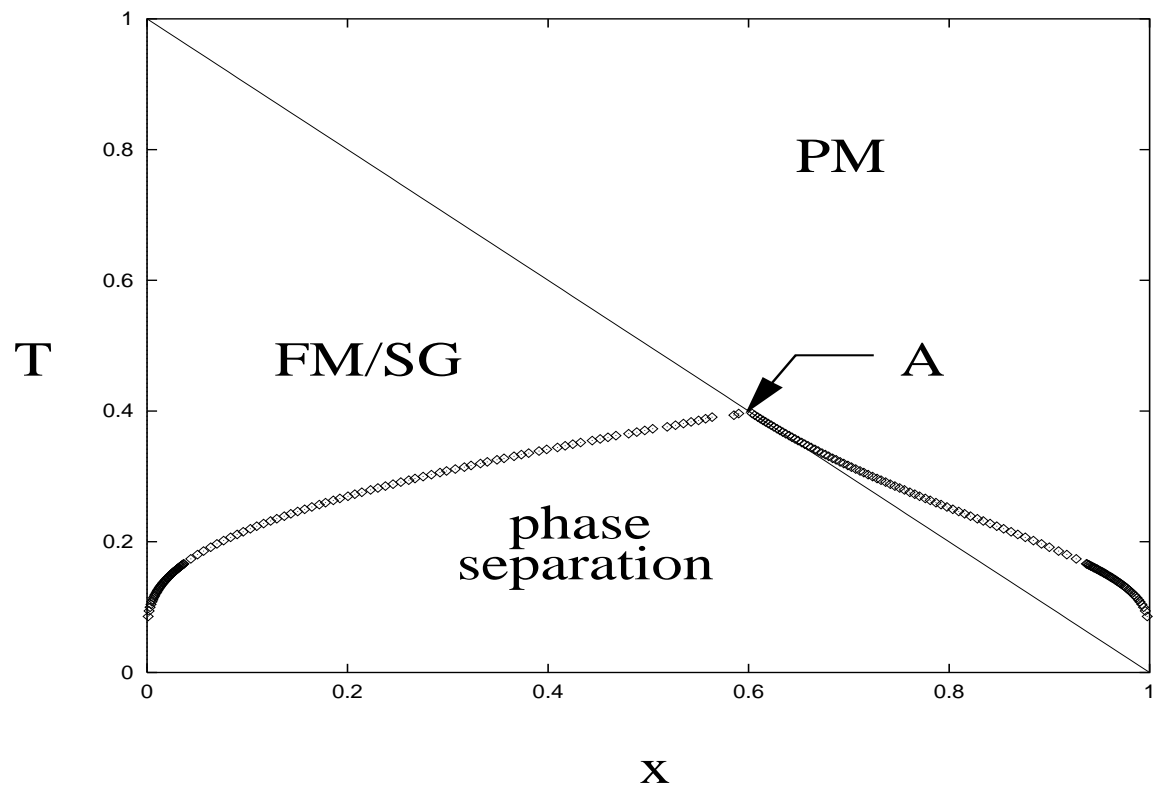

FIG. 1. The phase diagram of the (non-disordered) BEG model in the concentration/temperature-plane, i.e., $x-T$-plane, for the bilinear coupling $J_{0}=1.0$ and for weak quadrupolar coupling $K=0.16$. The thin line represents a transition of second order, whereas the diamonds indicate a transition of first order. FM/SG stands for ferromagnetic/spin glass phase,, $\mathrm{PM}$ is the paramagnetic phase; the point A indicates the tricritical point.

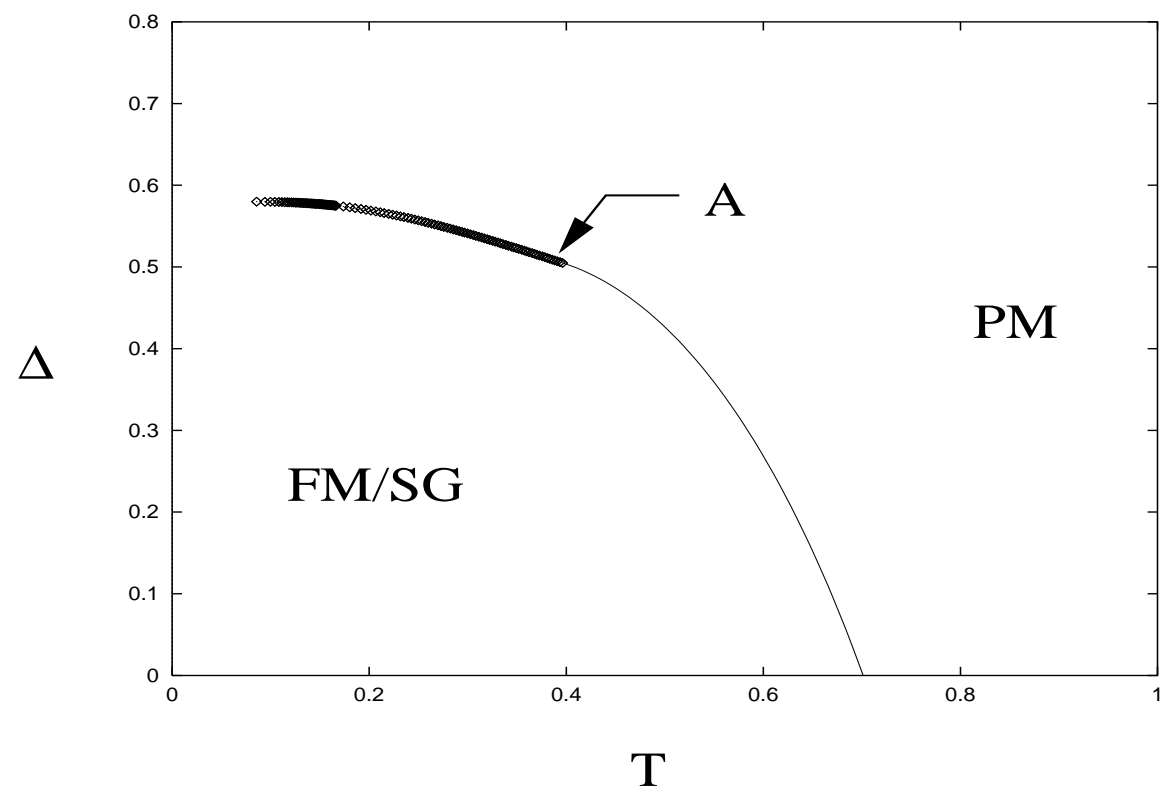


FIG. 2. The phase diagram of the (non-disordered) BEG model in the chemical potential/temperature-plane, i.e., $\Delta-T$-plane, for the bilinear coupling $J_{0}=1.0$ and for weak quadrupolar coupling $K=0.16$. The thin line represents a transition of second order, whereas the diamonds indicate a transition of first order.

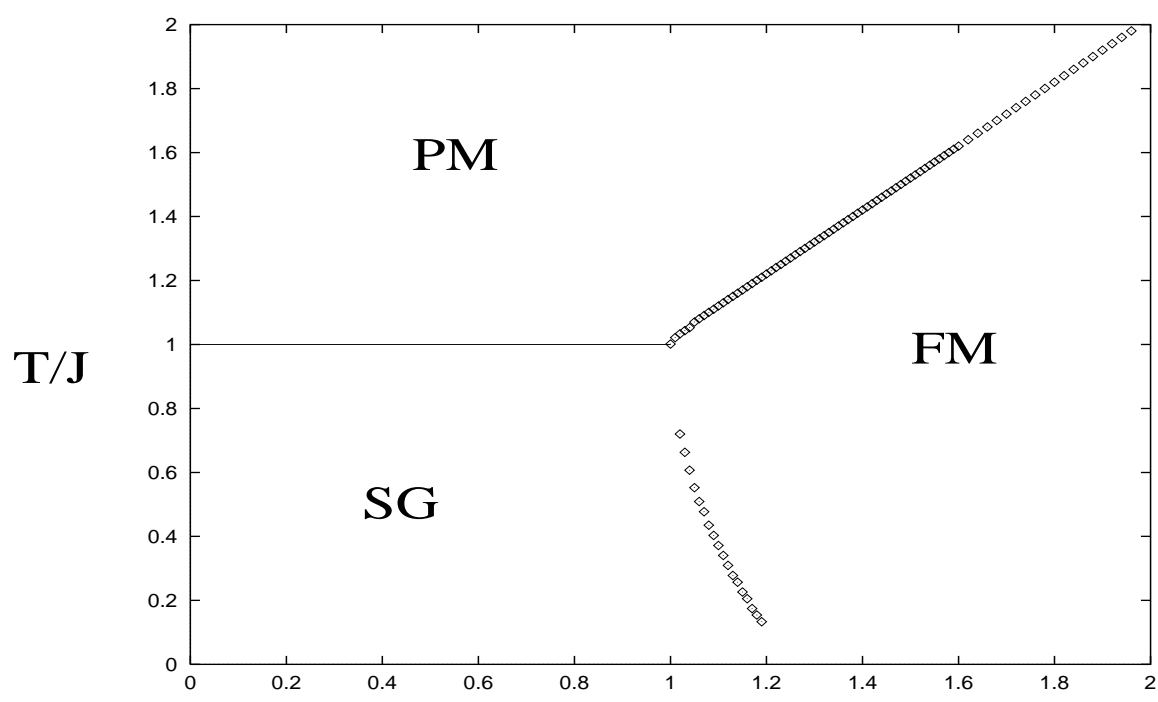

$\mathbf{J o} / \mathbf{J}$

FIG. 3. The phase diagram of the SK model in the ferromagnetic coupling/temperature-plane, i.e., $J_{0} / J-T$-plane. The lines shown are transitions of second order.

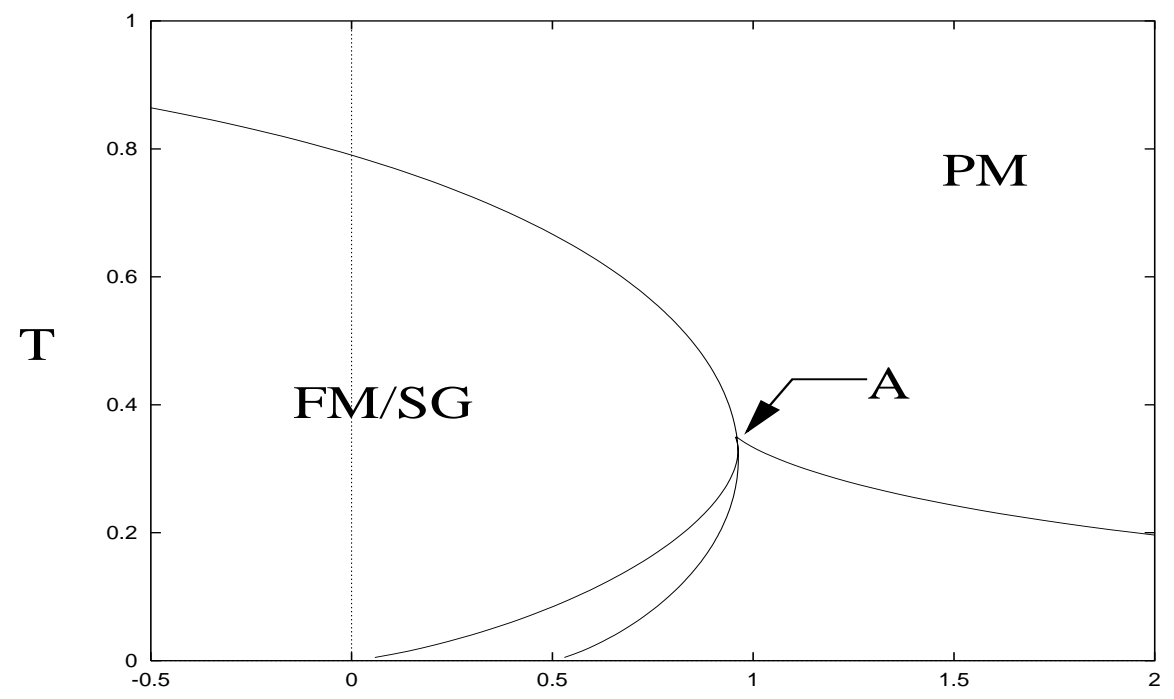

$\Delta$

FIG. 4. The phase boundary of the GS-model $\left(K=0.0\right.$ and $\left.J_{0}=0.0\right)$ in the chemical potential/temperature-plane, i.e., $\Delta-T$-plane. 


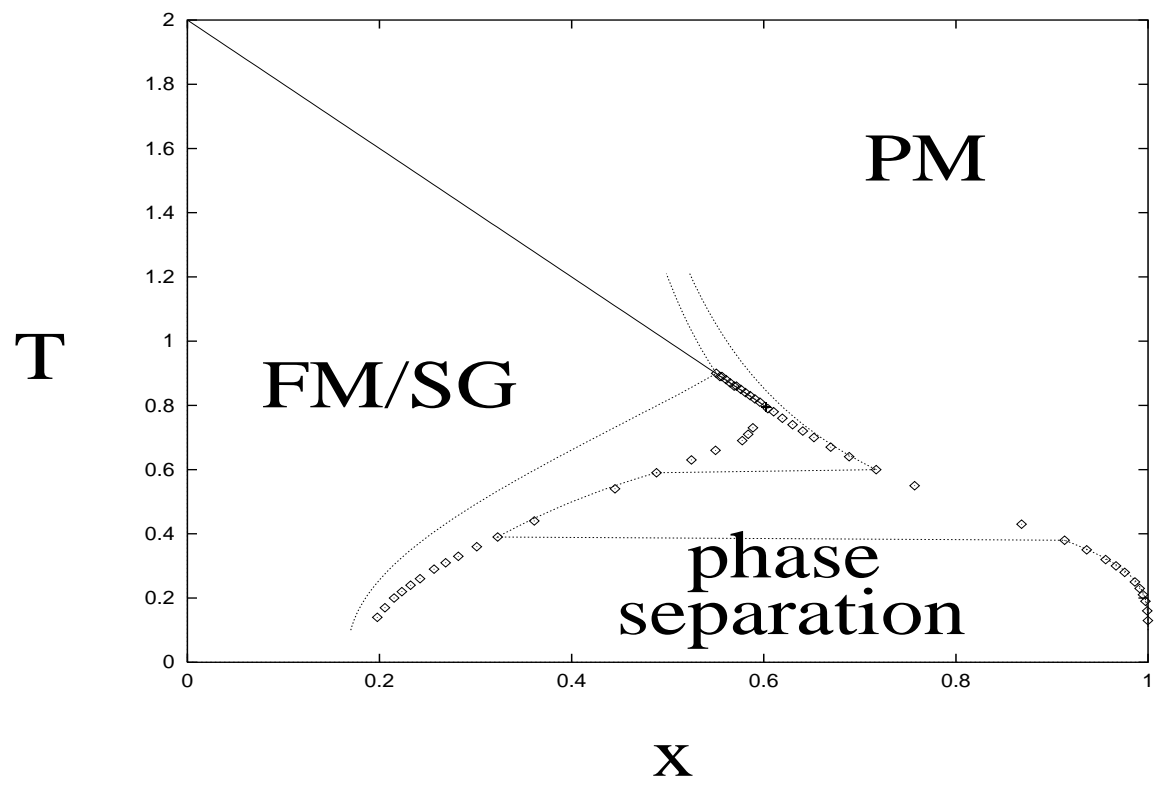

FIG. 5. The phase diagram of the disordered BEG-SK model in the $x-T$-plane for the bilinear couplings $J_{0}=2.0, J=1.0$ and for weak quadrupolar coupling, $K=0.16$. The thin line and the dense lying diamond symbols represent the transition of second order, whereas the sparse lying diamonds indicate the first order transition. The two dotted lines are the free energy minima for two different chemical potentials $(\Delta=0.6$ and 0.65$)$ and varying the temperature (from $T=0.0$ to $T=1.2)$.

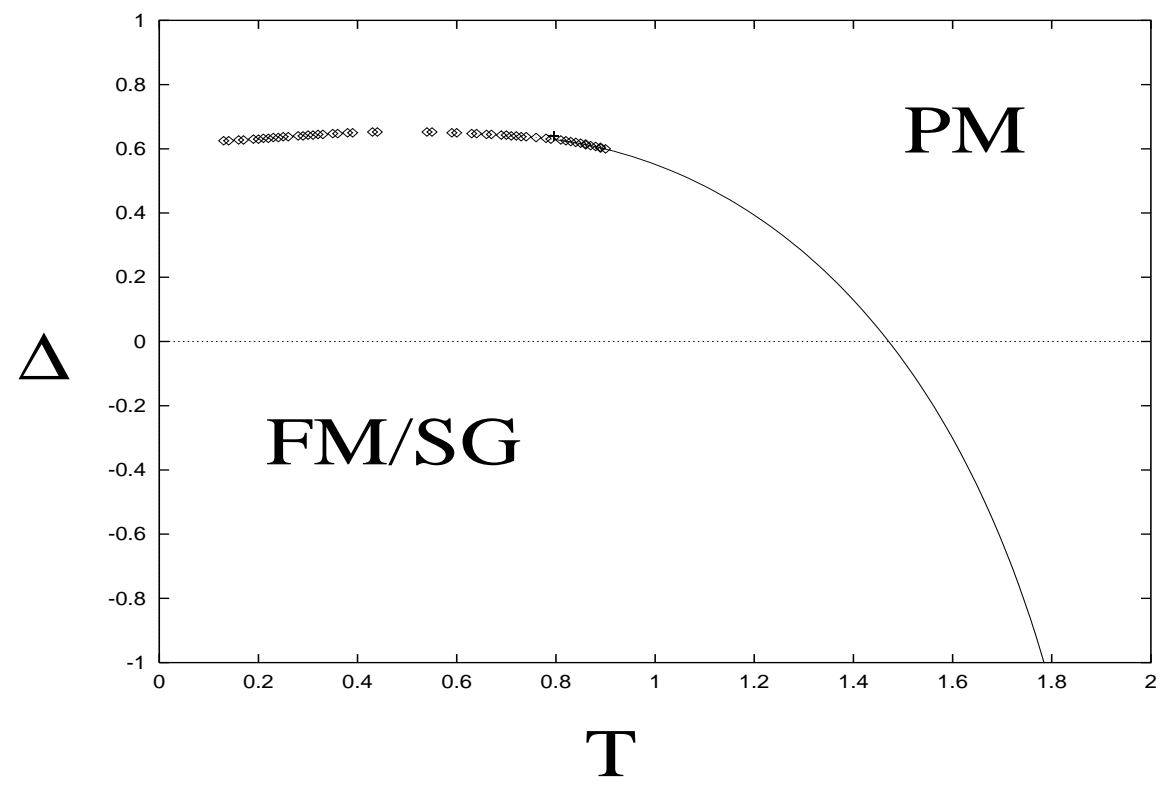


FIG. 6. The phase diagram of the disordered BEG-SK model in the $\Delta$ - $T$-section for the bilinear couplings $J_{0}=2.0, J=1.0$ and for weak quadrupolar coupling $K=0.16$. The thin line and the dense lying diamond symbols represent the second order transition, whereas the sparse diamonds indicate the transition of first order.

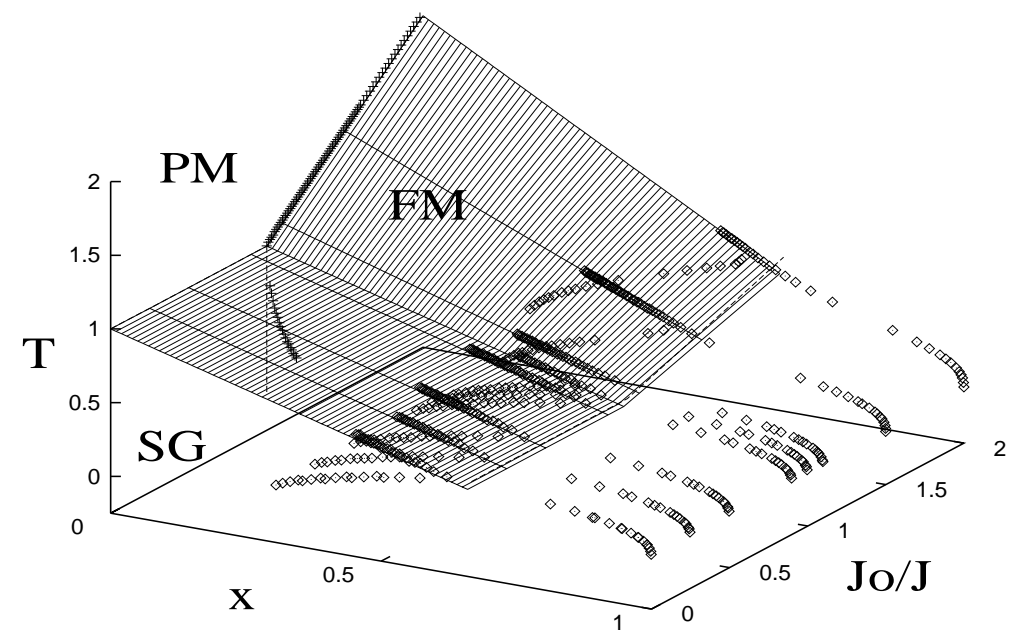

FIG. 7. The complete phase diagram of the disordered BEG-SK model, with the lines of stability and the line of tricritical temperature in the concentration/ferromagnetic coupling/temperature-representation, i.e., $x-J_{0} / J-T$-plane, for weak quadrupolar coupling $K=0.16$.

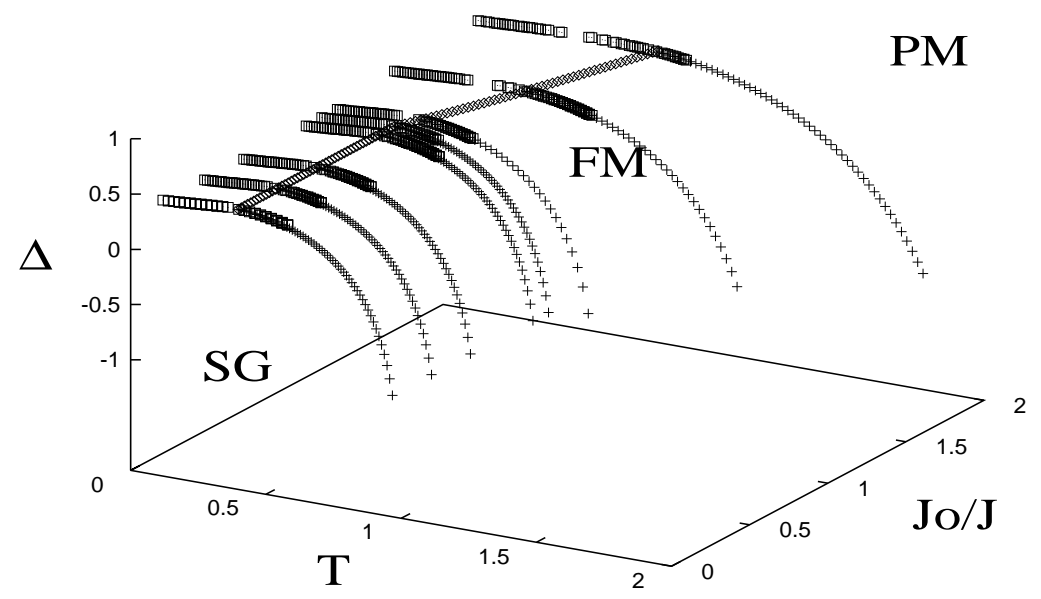


FIG. 8. The complete phase diagram of the disordered BEG-SK model, with the lines of stability and the line of tricritical chemical potential in the chemical potential/ferromagnetic coupling/temperature-representation, i.e., $\Delta-J_{0} / J-T$-plane, for weak quadrupolar coupling $K=0.16$

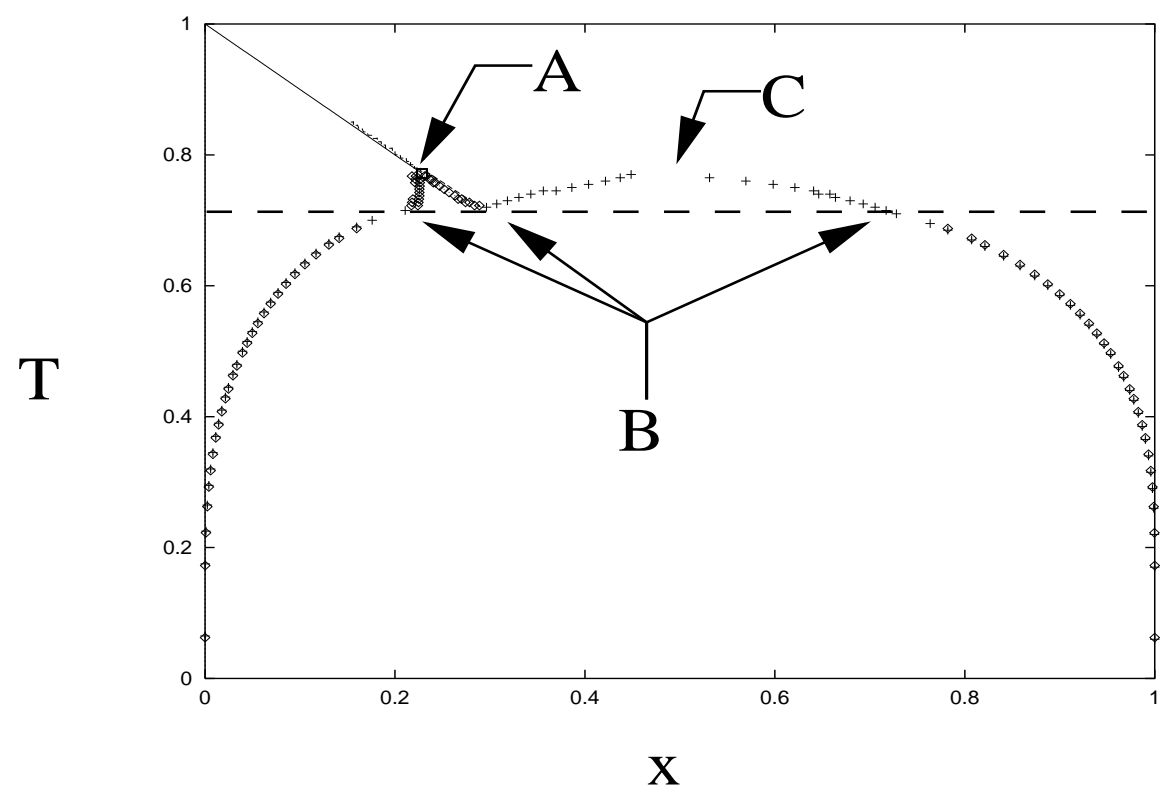

FIG. 9. The phase diagram of the non-disordered BEG model in the $x-T$-plane for the bilinear coupling $J=1.0$ and for a rather strong quadrupolar coupling, $K=2.88$. The point A indicates the tricritical point, the point $\mathrm{B}$ represents the triple point and point $\mathrm{C}$ is the critical end point.

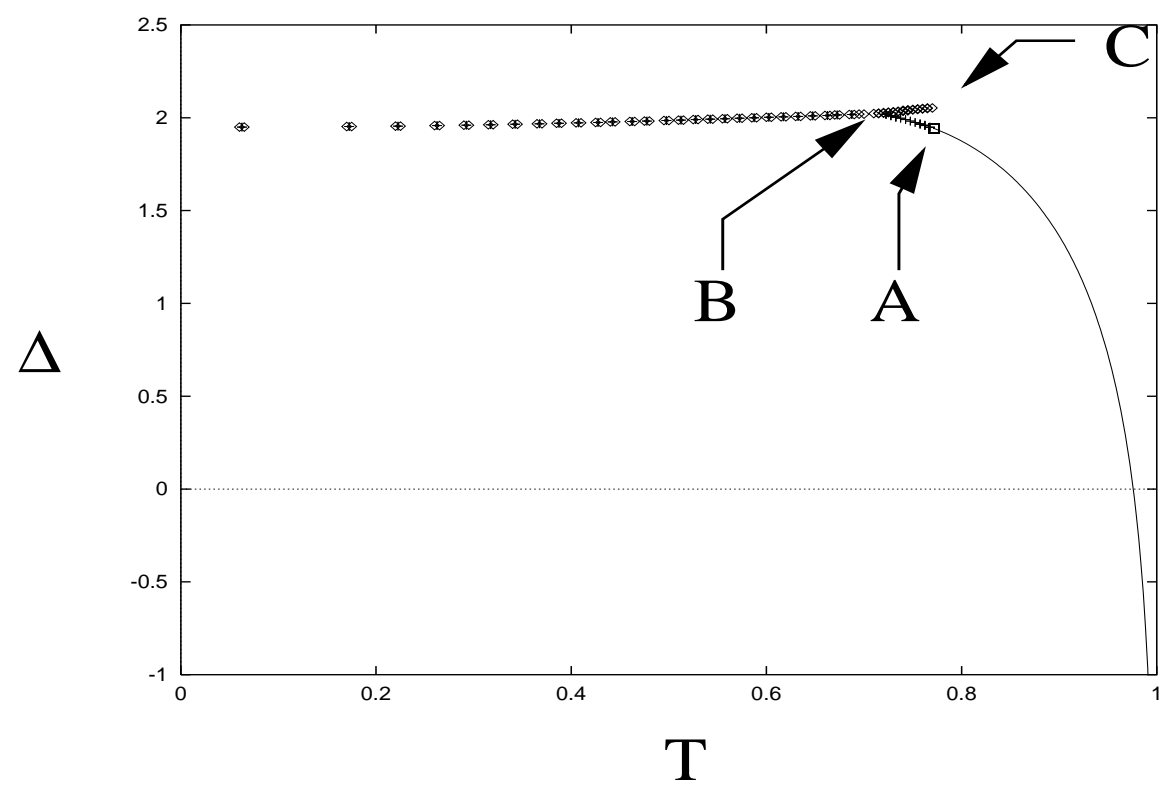


FIG. 10. The phase diagram of the non-disordered BEG model in the $\Delta-T$-section for the bilinear coupling $J=1.0$ and for a rather strong quadrupolar coupling $K=2.88$.

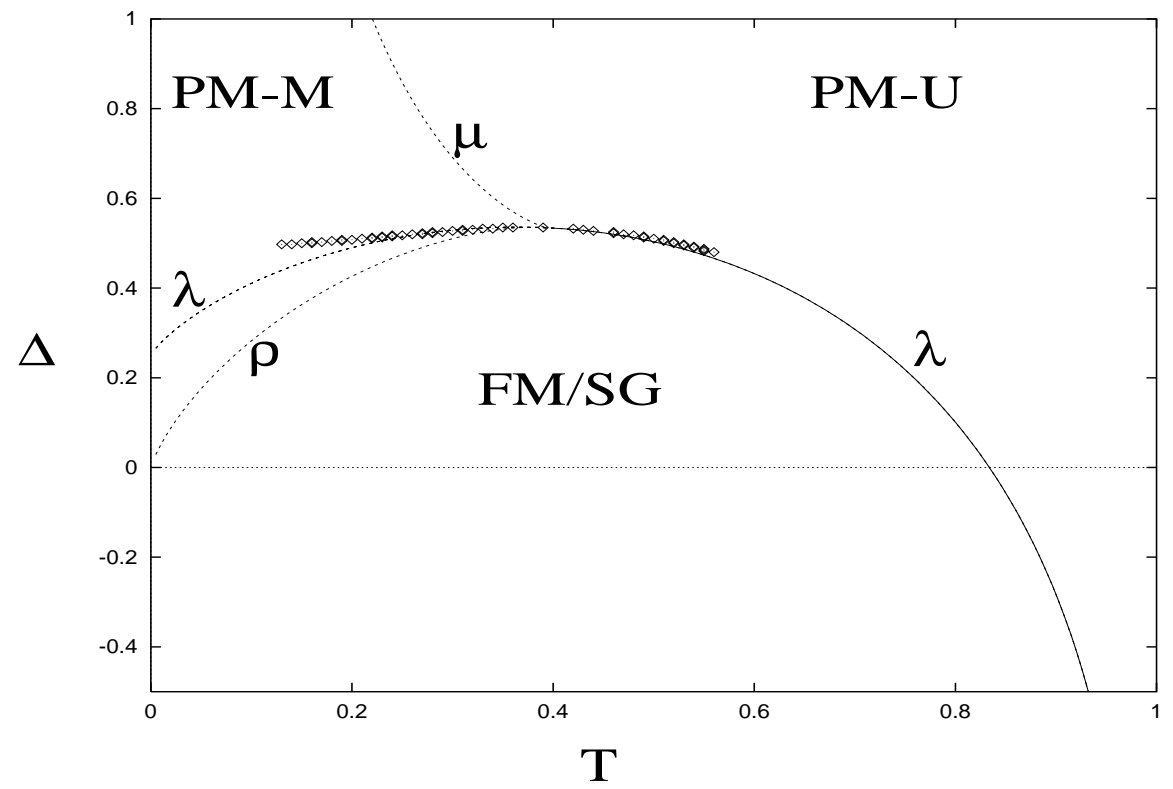

FIG. 11. The phase diagram of the disordered BEG-SK model, with the lines of stability in the chemical potential/temperature-representation, i.e., $\Delta-T$-plane, for weak quadrupolar coupling, $K=0.16$, and for the ferromagnetic coupling $J_{0} / J=2.0$. The symbols have the following meanings: PM-M is the paramagnetic phase with many free energy minima, PM-U is the paramagnetic phase with an unique free energy minimum, FM/SG the ferromagnetic or eventually spin glas phase, $\mu$ gives the positive sign branch and $\varrho$ the negative sign branch of equation (22); $\lambda$ is the second order transition line. 